\title{
Catalytically active bispecific antibodies - new biochemical markers of HIV/AIDS
}

Svetlana Baranova

ICBFM SB RAS, Novosibirsk, Russia

swb@niboch.nsc.ru

\author{
Sergey Sedykh \\ ICBFM SB RAS, Novosibirsk, Russia \\ sedyh@niboch.nsc.ru
}

\author{
Georgy Nevinsky \\ ICBFM SB RAS, Novosibirsk, Russia \\ nevinsky@niboch.nsc.ru
}

\begin{abstract}
Prognosis of the HIV/AIDS infection is an important problem of molecular medicine. Since there were described a number of changes similar to autoimmune processes in patients with HIV/AIDS, such changes might be new markers for disease prognosis.
\end{abstract}

\section{Keywords - exosomes, milk, microRNA}

\section{Motivation and aim}

HIV-infection is a viral disease, causing an immune deficiency. In patients with HIV/AIDS there were described a number of changes similar to autoimmune processes. An important problem of HIV/AIDS research is the prognosis of infection. As it was previously shown, autoimmune processes damage the nervous systems, and one of the markers of these processes are catalytic antibodies and bispecific antibodies. Such antibodies in vitro can hydrolyze neurospecific substrates.

\section{Methods}

Antibodies hydrolyzing neurospecific substrates were described in the blood of healthy donors, patients with systemic lupus erythematosus (SLE) and multiple sclerosis
(MS). Proteolytic IgGs hydrolyze myelin basic protein and oligodendrocytic peptide. We have shown that sera of HIV/AIDS, SLE and MS patients contain autoantibodies against histones and myelin basic protein.

\section{Results}

Here we show the results of research of natural bispecific catalytically active antibodies isolated from the blood of HIV/AIDS patients. Also, we compared the level of the protease activity of antibodies with the stage and characteristics of the pathological process. Interestingly, the pathological processes in HIV/AIDS are similar with such in MS and SLE. The development of new methods for diagnosing the patient's condition, the effectiveness of therapy, and also predicting disease outcome in the future can be used for personalized therapy and improving the patient's quality of life.

\section{ACKNOWLEDGMENT}

The research was supported by Grant of RFBR № 20-3470115 to Sergey Sedykh. 